\title{
Beckett et le nouveau néant
}

\author{
Geneviève Hamel \\ Collège André-Laurendeau
}

\begin{abstract}
va-et-vient dans l'ombre, de l'ombre intérieure à l'ombre extérieure

du soi impénétrable au non-soi impénétrable en passant par ni l'un ni l'autre

Beckett, 2004 [1980], p. 79
\end{abstract}

Il n'y a rien sur l'écran de l'ordinateur, qu'un fond noir d'un noir infini. Aucune image ne viendra s'y greffer durant trois minutes dix-sept secondes, hormis quelques changements abrupts ou très lents vers un fond blanc tout aussi infini. Aucune image, mais des sons en alternance ou en superposition : le bruit d'une haute fréquence de plus en plus fort et, dès lors, de plus en plus 
agressant, celui d'un tic-tac semblable à un métronome, le tout par moments doublé d'un grésillement. Puis, enfin, le silence. Le spectateur en doutera peut-être, mais il a pourtant regardé une vidéo, une forme qui se définit habituellement par la transmission d'images en mouvement. En ligne depuis 2009 sur YouTube, l'œuvre en question est conçue par un dénommé Noisesmith et s'intitule For Company. Loin d'être virale, elle n'avait été vue qu'à une centaine de reprises plus de cinq ans après sa première diffusion.

Que penser de cette œuvre qui ne représente, à peu de chose près, que du néant? Quel lien cette vidéo expérimentale peut-elle entretenir avec la littérature? L'artiste offre un début de réponse dans la description qui accompagne sa création, précisant qu'elle est «about the novel Company, by Samuel Beckett ». Ainsi, le titre For Company laisse sous-entendre que la vidéo serait une sorte d'hommage et invite à explorer le rapport de filiation entre les deux œuvres. Sans prétendre être une adaptation à proprement parler, For Company soulève de nombreuses réflexions quant à une pratique parfois controversée: la récupération de l'œuvre beckettienne à travers les médias d'aujourd'hui. Choisie ici parce qu'elle constitue un cas extrême, l'œuvre de Noisesmith servira de point de départ afin de réexaminer d'abord le rapport qu'entretenait Beckett à la fois avec le processus de l'adaptation, que son œuvre inspirait depuis bien avant sa mort, et avec les médias, qu'il a lui-même utilisés afin de créer des œuvres autres que littéraires. Une fois mis de côté les présupposés habituels entourant la légitimité ou la fidélité de l'adaptation, le regard posé sur l'œuvre de Noisesmith ouvre de nouvelles perspectives. 


\section{Beckett et les médias}

Lorsque l'on se penche sur les œuvres médiatiques actuelles inspirées de l'œuvre beckettienne, la question qui revient le plus souvent est celle de Jonathan Kalb: «Do directors and producers serve his legacy when they reach out to the benighted masses and compromise with them to try to win him millions more? » D'autres critiques et intellectuels soulèvent des questions encore plus drastiques ou subjectives, se demandant si les artistes ont le droit d'adapter les œuvres de Beckett ou encore si l'auteur aurait lui-même approuvé un tel projet. Ce faisant, ces critiques semblent oublier que si Beckett a - de son vivant - refusé plusieurs projets d'adaptation, il en a aussi approuvé d'autres, allant même jusqu'à donner carte blanche à certains réalisateurs. Plus encore, certaines adaptations ont eu un effet direct sur le travail de l'auteur. C'est le cas, par exemple, de Not I, adapté du théâtre à la télévision par la BBC en 1977. Dans la version originale, une Bouche faiblement éclairée crache une logorrhée quasi ininterrompue en direction d'un Auditeur debout sur scène, dont les seules interventions silencieuses correspondent à «une sorte de haussement des bras dans un mouvement fait de blâme et de pitié impuissante» (Beckett, 1996, p. 95). Lorsqu'il adapte la pièce pour le petit écran, Tristram Powell propose de supprimer complètement le personnage de l'Auditeur et de présenter un extrême gros plan de la Bouche du début à la fin. $\mathrm{Si}$, aujourd'hui, l'œuvre de Noisesmith fait vivre à ses spectateurs une expérience inusitée et difficile à supporter, on peut concevoir que l'adaptation de Not I, réalisée il y a plus de 30 ans, a dû donner des sueurs froides aux téléspectateurs britanniques sagement assis dans leur salon... 
Cet exemple, anodin en apparence, permet d'élargir la perspective avec laquelle les adaptations actuelles sont habituellement accueillies. D’abord, il déconstruit l'idée reçue selon laquelle Beckett était totalement opposé aux adaptations de son œuvre. Il le fut parfois, regretta aussi d'avoir donné son accord à certaines d'entre elles, mais pas systématiquement. Pourquoi? Plus que la réputation d'un réalisateur (l'écrivain a refusé le projet du très reconnu Ingmar Bergman), ce sont plutôt les capacités du médium envisagé à servir le propos de son œuvre et la vision esthétique que porte celle-ci qui semblent importantes. Car le passage d'un médium à un autre soulève de nombreuses considérations qui vont au-delà du respect du texte. L'adaptation de Not I le prouve d'emblée, puisque le réalisateur a balayé du revers de la main non seulement l'indication adressée à l'acteur, mais le jeu et l'existence même de cet acteur sur scène. Pourquoi Beckett a-til permis une telle modification? Selon Enoch Brater, lorsque l'équipe de la $\mathrm{BBC}$ proposa de transformer à ce point la mise en scène originale, "[i]ntrigued by the idea of a close-up, the playwright, always open to the adventure of exploring what a given medium can be made to do, agreed » (Brater, 1987, p. 35). Non seulement sera-t-il satisfait du résultat - affirmant que c'était «nettement la meilleure façon de rendre cette œuvre » (Beckett, cité par Bishop, 1990, p. 385) -, mais il transformera par la suite sa propre mise en scène de la pièce. Dans la version théâtrale corrigée par Beckett après l'adaptation télévisuelle, l'Auditeur disparaît, permettant ainsi de concentrer le regard des spectateurs sur la Bouche, tout comme l'extrême gros plan choisi lors de l'adaptation emprisonne celui des téléspectateurs durant un peu plus de dix minutes. 
Ce choix rappelle également que Beckett accordait une grande importance au langage propre à chaque médium. Dès le début des années 1960 et jusqu'en 1982, l'auteur crée lui-même des œuvres pour la radio, le cinéma ou la télévision. En ellesmêmes, ces œuvres montrent l'intérêt de Beckett envers les médias de son époque. L'utilisation qu'il en fait révèle au moins trois choses importantes : 1) peu importe le langage qu'il utilise, Beckett cherche à en souligner les failles, en les épuisant et en les mettant à nu; 2) comparativement à la littérature, ces médias lui permettent d'aller plus loin dans la déréalisation du sujet et dans le processus d'abstrahisation ${ }^{1}$ qu'il tente $^{\prime}$ constamment de mettre en œuvre; et 3) les allers et retours entre ces médias font évoluer sa vision esthétique, où l'image et la fragmentation de celle-ci prennent de plus en plus de place. Le passage de Not I à la télévision est un parfait exemple d'une telle transformation. Ainsi, plutôt que de se pencher sur la légitimité des adaptations, ne serait-il pas plus pertinent de s'attarder plus sérieusement à leur potentiel esthétique, c'est-àdire leur capacité à rendre hommage non seulement à l'œuvre originale, mais aussi et peut-être surtout à communiquer ou à poursuivre cette démarche artistique qui va bien au-delà des mots? Dans Samuel Beckett's Plays on Film and Television, Graley Herren affirme que «[r]ather than judging screen adaptations by an exacting standard of fidelity, we scholars need to train our critical ears to appreciate this different

1 L'abstrahisation s'apparente, selon Max Bense, au processus d'abstraction dans les arts plastiques. Il remarque que «le paradoxe de la prose beckettienne réside dans le fait qu'elle vise en permanence à annuler le processus esthétique, c'est-à-dire la formation des signes, le mouvement expressif ou bien le mouvement significatif » (2007, p. 230) et qu'il « s'agit du plus haut degré de non-identité de la chose et de la signification » (2007, p. 231). 
music » (2007, p. 196). Ce conseil se révèle particulièrement judicieux pour qui souhaite mieux saisir l'œuvre de Noisesmith dont il est question ici.

\section{Nouveau néant, nouvelle perspective}

Comment le spectateur réagit-il devant une œuvre telle que For Company? Les quelques dizaines de «vues » qu'elle a suscitées en cinq ans montrent tout au moins qu'il ne s'agit pas là d'une vidéo virale. Par conséquent, ni l'inquiétude des uns (qui ont peur de voir l'œuvre originale «mal servie» par de nouvelles interprétations), ni l'espoir des autres (qui souhaiteraient que les adaptations attirent l'attention d'un nouveau public sur l'œuvre de Beckett) n'entrent en jeu ici. En supposant que le spectateur ne connaisse rien à l'œuvre de Beckett, que peut-il comprendre ou conclure d'une telle adaptation? Et s'il connaît l'œuvre originale, comment réagira-t-il devant cette absence totale de mots? Car ce n'est pas seulement un personnage ou un segment du texte qui aurait été supprimé ou altéré, mais bien l'entièreté du texte qui a disparu derrière cet écran noir.

Il serait possible, comme pour toute adaptation, de procéder d'abord à une analyse comparative entre les deux œuvres en s'appuyant, comme c'est généralement le cas, sur le contenu respectif de chaque version. Quelles analogies peut-on voir entre le texte Compagnie et la vidéo For Company? Le récit original, écrit en anglais (Company) entre les années 1977 et 1979, s'ouvre comme suit: "Une voix parvient à quelqu'un dans le noir. Imaginer. Une voix parvient à quelqu'un sur le dos dans le noir. Le dos pour ne nommer que lui le lui dit et la façon dont change le noir quand il rouvre les yeux et encore quand il 
les referme.» (Beckett, 2004 [1980], p. 7) Environ trente ans après L'Innommable, Beckett poursuit son travail de sape, cette déconstruction du langage qui va souvent de pair avec la déréalisation du sujet. Ici, une voix s'adresse à un " entendeur », nommé ainsi puisqu'il est condamné à la seule action d'entendre, presque incapable de bouger (à la limite, à peine peut-il ramper difficilement) et surtout incapable de parler. Sa position, ses gestes, sa respiration, l'état de son esprit et même jusqu'à ses souvenirs lui sont dictés par la voix. Le choix du fond noir et de l'absence d'image qui caractérise For Company pourrait être expliqué par cet incipit: «Une voix parvient à quelqu'un dans le noir. Imaginer.» (p.7) Plus loin, l'auteur précise que le personnage à qui parvient la voix, existant à peine, est plongé dans un "noir immensurable. Sans bornes. » (p.45) Difficile de ne pas croire que c'est dans ce même noir que Noisesmith souhaite plonger ses spectateurs. Et la voix qui parvient à l'entendeur correspondrait-elle alors au bruit strident? Après tout, l'auteur indique que

[1]a voix à elle seule tient compagnie mais insuffisamment. Son effet sur l'entendeur est un complément nécessaire. Ne seraitce que sous la forme du vague sentiment d'incertitude et de gêne susmentionné. Mais même mise à part la question de compagnie il est évident qu'un tel effet s'impose. Car s'il devait seulement entendre la voix et qu'elle n'ait pas plus d'effet sur lui qu'une parole en bantou ou en erse ne ferait-elle pas aussi bien de se taire? À moins qu'elle ne vise en tant que bruit à l'état pur à mettre au supplice un affamé de silence. (p. 11)

N'est-ce pas là, en quelque sorte, l'effet que ce bruit strident a sur le spectateur? Un supplice, une torture si insupportable qu'elle fait rapidement et ardemment espérer son ultime opposé: le silence. Peut-on expliquer les éclairs blancs qui apparaissent furtivement dans l'œuvre de Noisesmith? Bien sûr, 
celui qui se penche sur le texte de Beckett se dira qu'il y a sans doute un lien à faire avec la lueur que perçoit l'entendeur lorsque parle la voix: "La voix émet une lueur. Le noir s'éclaircit le temps qu'elle parle. S'épaissit quand elle reflue. S'éclaircit quand elle revient à son faible maximum. Se rétablit quand elle se tait. » (p. 24) Les tentatives d'analogies entre le texte et la création de Noisesmith pourraient être encore nombreuses. Toutefois, malgré ces quelques clins d'œil qui renvoient à l'œuvre originale (non sans une certaine gymnastique mentale), la lecture de Compagnie risque de rendre l'analyse de For Company bien décevante au regard de qui chercherait une simple transposition de l'œuvre. Car le lecteur découvre très rapidement que la voix ne sert pas qu'à torturer son entendeur : elle lui «égrène un passé. Question aussi par moments d'un présent et plus rarement d'un avenir » (p.8). Et cette histoire racontée par la voix, sans que l'entendeur ne soit convaincu qu'elle est la sienne, est faite de détails si précis et si nombreux qu'il serait impossible de ne pas être assailli par les images qu'ils provoquent. Des images, n'estce pas précisément ce qu'un cinéaste aurait été tenté de représenter? D’ailleurs, Stéphane Inkel « [d]istingue deux types d'images, dans Compagnie. Les images de la Voix, supposées rendre compte de la vie passée du corps, de même que l'image de cette Voix, qui émet une faible lueur lorsqu'elle parle à "quelqu'un sur le dos dans le noir". Une image du geste de la Voix. » (2003, p. 109) Dans son hommage, Noisesmith parvient peut-être bien à concrétiser ce geste de la Voix, grâce aux mouvements entre le noir et les quelques lueurs blanches. Mais il ne rend aucunement compte du premier type d'images proposé par Inkel, puisque les souvenirs racontés à l'Entendeur se sont complètement évaporés. Par conséquent, force est 
d'admettre que l'étude comparative des deux œuvres soulève, malgré les quelques analogies possibles, un certain nombre de failles. Pourquoi alors For Company mérite qu'on s'y attarde?

\section{Vers l'en-deçà du réel}

Intrigué par la démarche artistique de Noisesmith, et par conséquent par celle de Beckett, un spectateur pourrait se demander pourquoi cet artiste a voulu représenter trois minutes dix-sept secondes de noir entrecoupé d'éclairs blancs, avec en prime des sons hautement intrigants (pour ne pas dire insupportables). En y regardant de plus près, peut-être remarquerait-il que ce nom d'artiste, Noisesmith, souligne en quelque sorte l'importance du bruit dans son œuvre (puisque ce pseudonyme signifierait à peu près "artisan du bruit»). Mais, surtout, ce qui gagne à être regardé de plus près est la courte description indiquant qu'il s'agit d'une vidéo faite d'images-synthèses à partir d'un logiciel libre de montage audio-vidéo nommé Cinelerra. À première vue, particulièrement pour les néophytes en la matière, cela semble un détail sans importance. Or, lorsqu'un artiste choisit de créer une vidéo ou un film à partir d'images synthèses, c'est qu'il ne peut ou ne veut pas créer à partir d'images réelles (celles de phénomènes existants, vivants, mouvants, qu'il se contenterait alors de filmer). Ici, on se trouve donc devant quelque chose de totalement irréel. Et cet irréel, plutôt que d'aller au-delà des limites du réel (ce que font tous les réalisateurs de films d'animation ou de science-fiction en créant des mondes surnaturels), va en deçà du réel. Ce serait donc ce que l'on pourrait appeler de la non-existence à l'état pur. 
Doit-on revenir au texte original pour comprendre que Noisesmith a peut-être essayé d'atteindre exactement ce que Beckett tentait de représenter, avec les moyens propres au langage qu'il a choisi d'utiliser? Sous forme de texte, Compagnie met en scène ce que Jeannette Den Toonder décrit comme une «situation extrême d'incommunication» (1996, p.146). Autrement dit, l'auteur tenterait de dire l'impossibilité de dire. Mais pour le dire, il doit créer, et ce qu'il crée doit exprimer le fait qu'il est impossible de représenter le réel. C'est précisément ce que Geneviève Serreau exprimait lorsqu'elle écrivait au sujet de Compagnie que le texte parvient à

nous livrer un «modus scribendi», une sorte d'art poétique, en nous rendant immédiatement (et presque physiquement) perceptible la situation de l'écrivain par rapport à ce qu'il crée. Flottant entre être et néant, celui-là a établi sa demeure provisoire dans l'imaginaire, voué à épuiser en mots l'infini du temps et l'irréalité de l'espace, incapable de "tricher », c'est-àdire de faire semblant de raconter des histoires et d'inventer des personnages et d'y croire, alors que la solitude est son lot définitif, gloire et détresse mêlées. (1980)

Car c'est bien ainsi que se termine le texte de Beckett. Le personnage git toujours dans le noir et la voix lui dit ceci :

Mais le visage renversé pour de bon peinera en vain sur ta fable. Jusqu'à ce qu'enfin tu entendes comme quoi les mots touchent à leur fin. Avec chaque mot inane plus près du dernier. Et avec eux la fable. La fable d'un autre avec toi dans le noir. La fable de toi fabulant d'un autre avec toi dans le noir. Et comme quoi mieux vaut tout compte fait peine perdu et toi tel que toujours.

Seul. (Beckett, 2004 [1980], p. 87-88)

Et si le grand projet de Beckett était d'utiliser les mots pour tenter de mieux les trouer, quitte à montrer le vide qui se cache 
derrière (comme il l'écrivait dans la fameuse Lettre allemande ${ }^{2}$ ), Noisesmith aura peut-être alors fait de même avec les images. Et c'est justement là la toute-puissance du bruit, ce matériau au cœur des deux œuvres dont il est ici question. C'est semble-t-il encore une fois Stéphane Inkel qui le dit avec le plus de justesse: "La transcendance de la Voix ne représente plus, dans Compagnie, une instance d'aliénation, mais au contraire cette part d'invention qui échoit à l'image. La puissance de l'image est indissociable, chez Beckett, d'une voix qui en prépare le surgissement. » (2003, p. 110).

\section{Pour finir encore}

Reste bien évidemment la possibilité que le spectateur soit tout simplement énervé par le bruit insupportable ou dégoûté par l'absence de signification (ou simplement de divertissement) et qu'il en conclue que Noisesmith (et peut-être Beckett aussi) n'a décidément pas autant de talent que Lady Gaga... Mais, si le spectateur s'impatiente, s'injurie et s'énerve, peut-être est-ce ce qu'il a de mieux à faire? En effet, comme Beckett le précisait à l'interprète de Not $I$, à qui il demandait de réciter son texte toujours plus vite: «Mon intention n'est pas de m'adresser à leur intellect. Je veux que cela leur tape sur les nerfs. » (Beckett, cité par Fehsenfeld, 1990, p. 366)

\footnotetext{
2 Adressée à son ami allemand Axel Kaun le 9 juillet 1937, cette lettre est considérée par de nombreux critiques beckettiens comme l'ébauche du projet esthétique de Beckett. L'auteur écrit entre autres ceci : "Il m'est en fait de plus en plus difficile, absurde même, d'écrire dans un anglais officiel. Et ma propre langue m'apparaît de plus en plus comme un voile qu'il faut déchirer afin d'atteindre les choses cachées derrière (ou le rien caché derrière). » (2007 [[1937]1983], p. 15)
} 


\section{Bibliographie}

BECKETT, Samuel. (2004 [1980]), Compagnie, version française de Company, traduit par l'auteur, Paris, Minuit.

-. (1996 [1973]), Pas moi, dans Oh les beaux jours, suivi de Pas moi, version française de Not I, traduit par l'auteur, Paris, Minuit.

\section{Pièces radiophoniques}

BECKETT, Samuel. (1990 [1956]), Tous ceux qui tombent, version française de All that fall, traduit par Rober PINGET, Paris, Minuit.

—. (1997 [1959]), "Cendres», version française de Embers, traduit par Robert PINGET et l'auteur, dans La Dernière Bande suivi de Cendres, Paris, Minuit, p. 35-72.

—. (2009 [1962]), «Paroles et musique », dans Comédie et actes divers, Paris, Minuit, p. 61-78.

—. (2009 [1963]), "Cascando», dans Comédie et actes divers, Paris, Minuit, p. 45-60.

—. (1995 [1973]), "Esquisse radiophonique», dans Pas, suivi de Quatre esquisses, Paris, Minuit, p. 87-97.

—. (1995 [1975]), "Pochade radiophonique» dans Pas, suivi de Quatre esquisses, Paris, Minuit, p. 63-85. 


\section{Pièces télévisuelles}

-. (2009 [1967]), «Dis Joe», version française de Eh Joe, traduit par l'auteur, dans Comédie et actes divers, Paris, Minuit, p. 79-92.

—. (2002 [1976]), "Trio du Fantôme», version française de Ghost Trio, traduit de l'anglais par Édith Fournier, dans Quad et autres pièces pour la télévision, Paris, Minuit, p. 17-36.

—. (2002 [1977]), « ... que nuages... », version française de ...but the clouds..., traduit de l'anglais par Édith Fournier, dans Quad et autres pièces pour la télévision, Paris, Minuit, p. 37-48.

—. (2002 [1984]), "Nacht und Träume », version française de Nacht und Träume, traduit de l'anglais par Édith Fournier, dans Quad et autres pièces pour la télévision, Paris, Minuit, p. 49-54.

—. (2002 [1984]), "Quad», version française de Quad, traduit de l'anglais par Édith Fournier, dans Quad et autres pièces pour la télévision, Paris, Minuit, p. 7-15.

\section{Films}

BECKETT, Samuel. (2009 [1967]), "Film », version française de Film, traduit de l'anglais par l'auteur, dans Comédie et actes divers, Paris, Minuit, p. 111-134.

—. (2007 [[1937] 1983]), "La lettre allemande », dans Objet Beckett, Paris, Éditions du Centre Pompidou, p. 14-16. 


\section{Adaptations}

NoISESMITH. (2009), For Company, $<$ https://www.youtube.com/watch?v=l09oSUwQdVE $>$.

PowEll, Tristram. (1977), Not I, dans le cadre du programme Shades, Angleterre, co-production BBC TV-Reiner Moritz, $60 \mathrm{~min}$, $<$ https://www.youtube.com/watch?v=M4LDwfKxr-M>.

\section{Corpus critique}

BENSE, Max. (2007), Aesthetica, Introduction à la nouvelle esthétique, traduit de l'allemand par Judith Yacar, Paris, Éditions du Cerf.

Bishop, Tom. (1990), «Transposition pour la télévision: transmutations des œuvres de Beckett», Revue d'esthétique, numéro hors série dir. par Mikel Dufrenne et Olivier d'Allonnes, Paris, Éditions Jean-Michel Place.

BRATER, Enoch. (1987), "Shades for Film and Video », dans Beyond Minimalism, Beckett's late style in the theater, New York, Oxford University Press, p. 74-111.

DEN ToOnder, Jeannette. (1996), «Compagnie: Chimère autobiographique et métatexte », dans Sjef HoupPERMANS (dir.), Beckett \& la psychanalyse, Samuel Beckett Today/ Aujourd'hui 5, Amsterdam, Rodopi, p. 143-153.

FEHSENFELD, Martha. (1990), « De la boîte hermétique au regard implacable: le champ de l'image va se rétrécissant dans l'œuvre théâtrale de Beckett », Revue d'esthétique, numéro hors série dir. par Mikel Dufrenne et Olivier d'Allonnes, Paris, Éditions Jean-Michel Place. 
Herren, Graley. (2007), Samuel Beckett's Plays on Film and Television, New York, Palgrave Macmillan.

INKEL, Stéphane. (2003), « Le murmure et la voix : Transcendance, politique et compagnie chez Samuel Beckett », dans Imaginaire et transcendance, Montréal, Observatoire de l'imaginaire contemporain, <http://oic.uqam.ca/fr/articles/le-murmure-etla-voix-transcendance-politique-et-compagnie-chez-samuelbeckett>, d'abord paru dans Anne Élaine CLICHE, Stéphane INKEL et Alexis LuSSIER (dir.), Montréal. Figura, Centre de recherche sur le texte et l'imaginaire, vol. 8.

KALB, Jonathan. (2006), «Stardust Melancholy. Does the filming of Samuel Beckett's complete works compromise his theatrical legacy?», Theatre Communications Group, <http://www.tcg.org/publications/at/2003/stardust.cfm>.

SERREAU, Geneviève. (1980), « Beckett ou la dignité. Le pouvoir de nous toucher jusqu'au sang », Le Nouvel Observateur, 10 mars, $<$ http://www.leseditionsdeminuit.fr/f/index.php?sp=liv\&livr e_id $=1519>$. 


\title{
Résumé
}

Le désir d'adapter l'œuvre de Beckett soulève autant l'enthousiasme des artistes que la résistance des puristes. De telles reprises sont-elles vraiment en mesure de transmettre la vision de l'auteur à un nouveau public ou sont-elles en train de malmener la création originale et la réflexion esthétique qu'elle porte? C'est à partir de cette question qu'on examine le plus étrange de ces objets, For Company, de Noisesmith, tel que diffusé sur YouTube. Une fois mis de côté les présupposés habituels entourant la légitimité ou la fidélité de l'adaptation, le regard posé sur ce cas extrême ouvre de nouvelles perspectives.

\begin{abstract}
The desire to adapt Beckett's work has always raised as much enthusiasm from the artist as it has generated resistance from the purist. Are these adaptations truly able to convey the author's vision to a new audience, or are they harming the original creation and the æsthetic reflection within? This interrogation will guide our observation of the strangest of these objects, For Company, by Noisesmith, as displayed on YouTube. Once set aside the usual assumptions surrounding the legitimacy or the fidelity of the adaptation, the study of this utmost case opens new perspectives.
\end{abstract}

